\title{
Multi-Element Fibre for Space-Division Multiplexed Transmission
}

\author{
S Jain*, T. C. May-Smith, V. J. F. Rancaño, P. Petropoulos, D. J. Richardson and J. K. Sahu \\ Optoelectronics Research Centre, University of Southampton, Southampton, SO17 1BJ, U. K. \\ *sj3g11@orc.soton.ac.uk
}

\begin{abstract}
We present a multi-element fibre (MEF) as a novel candidate for space-divisionmultiplexing of optical channels that require high density of data transfer. 3-MEFs, comprising three individual fibres in a common coating, have been fabricated and error-free transmission has been demonstrated.
\end{abstract}

\section{Introduction}

Various modulation and multiplexing schemes have been implemented in optical fibres to accommodate the exponential data traffic growth experienced in today's communication networks [1,2]. However, there is limited scope remaining for exploiting further single mode fibres (SMFs), since current techniques are already approaching the Shannon-limit [3]. Therefore, growing attention is now being focused on increasing the number of spatial channels in the fibre using space-division multiplexing (SDM) [4,5]. In recent years, multicore fibres (MCF) have emerged as potential candidates for increasing the data capacity per-fibre cable, and MCFs with as many as 19 cores have been reported [6]. However, the accessibility of cores in MCFs still remains an issue [6]. Multiplexing/ demultiplexing (MUX/DEMUX) devices based either on free-space or bulk type optics systems are used in conjunction with MCFs to allow the cores to be addressed separately. Free-space optics MUX/DEMUX devices are more effective; however, they require accurate alignment, leaving questions as to their practicality for deployment in real systems. Also, the cladding diameter in MCFs is restricted to about $225 \mu \mathrm{m}$ in order to maintain the same mechanical reliability level as SMF [7]. This criterion, along with the minimum pitch required for low crosstalk, restricts the effective area of cores $\left(\mathrm{A}_{\text {eff }}\right)$ and maximum number of cores that can be accommodated in an MCF design.

We present an alternative robust and practical approach to implement SDM using multielement fibres (MEFs), which comprise multiple fibres that are drawn and coated together in a common polymer coating. MEFs offer convenience in fibre handling, as each fibre can be accessed individually, simply by removing the polymer coating; conventional splicing to SMF components can then be used. A typical MEF preform assembly is shown in Fig. 1a and the drawn fibre with its various elements fanning out at the two ends is shown in Fig. $1 \mathrm{~b}$, illustrating key aspects of the technology.

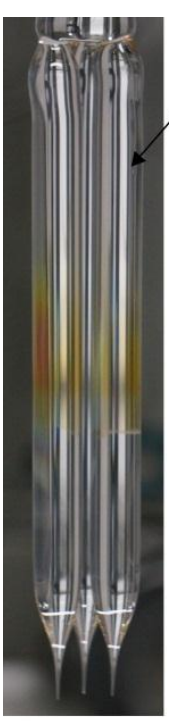

(a)

\section{Preform}

Assembly

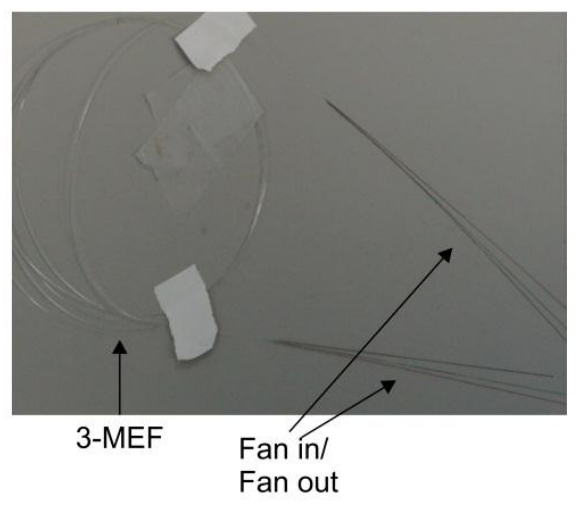

(b)
Fig. 1 a) 3-MEF preform assembly and b) 3-MEF with fibre-elements fanning out at each end

An additional advantage of the MEF configuration is that it no longer suffers from the overall diameter restriction of $225 \mu \mathrm{m}$ as compared to MCFs. MEF designs offer the potential to incorporate even more than 19 crosstalk-free SDM channels in a single fibre structure.

We have already demonstrated a core-pumped erbium (Er)-doped 7-MEF amplifier [8]. In this work, we have fabricated and performed the initial characterization of MEFs to demonstrate their potential for use in transmission. Here, the number of fibre elements was reduced from 7 to 3 (3-MEF) to facilitate the initial fabrication of $\mathrm{km}$-long lengths of MEF suitable for transmission experiments (we do not anticipate major challenges to scaling to larger element counts once we have optimized the basic approach). Also, a dual coating was applied to 
improve the overall fibre strength and bring it to the standards required for telecommunication deployment.

\section{Multi-Element Fibre (MEF)}

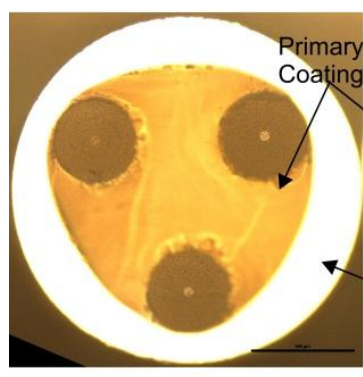

(a)

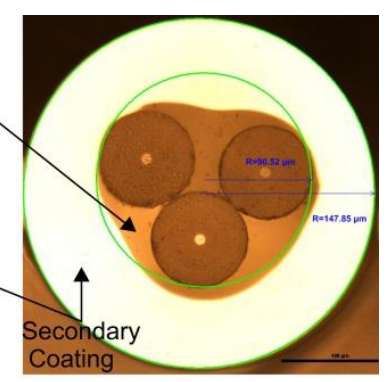

(b)
Fig.2. Microscope image of a) non-compact and, b) compact 3-MEF cross-section with dual polymer coating

Figures $2 a$ and $2 b$ show microscope images of the cross-section of two 3-MEFs that we fabricated; a non-compact and a compact one. The non-compact 3-MEF, with an element diameter of $80 \mu \mathrm{m}$, was first fabricated using clear fused quartz (CFQ) rods from Heraeus to test the strength of the fibres from the fabrication procedure. The fibre elements were coated together with a high-index dual polymer coating; Desotech DP-1004 and DS-2015 were used as the primary and secondary coating respectively. The 3-MEF was then proof-tested to measure the overall fibre strength. The strength test was performed on the fabricated 3MEFs using a standard industrial fibre rewind and proof testing instrument. In our case, the required weight corresponding to the proof test strength was calculated by multiplying the weight required for a single fibre with the same fibre-element diameter by the number of fibreelements in the MEF. The 3-MEF successfully passed the 400-kpsi strength test. Using the conditions obtained from this first draw, a 3MEF preform was assembled from a germanium (Ge)-doped silica preform with a step index difference $(\Delta n)$ of 0.0055 , and $9.5 \mathrm{~km}$ of 3-MEF was fabricated with fibre-element cladding and core diameters of $\sim 80 \mu \mathrm{m}$ and $8.5 \mu \mathrm{m}$ respectively. The overall coated diameter was $340 \mu \mathrm{m}$. The cut-off wavelength and dispersion at $1550 \mathrm{~nm}$ of each fibre-element was approximately $1250 \mathrm{~nm}$ and $18-\mathrm{ps} / \mathrm{nm} / \mathrm{km}$ respectively. The loss measured at $1550 \mathrm{~nm}$ with an Exfo-FTB-7300E optical time-domain reflectometer (OTDR) was $0.6 \mathrm{~dB} / \mathrm{km}$. Similar loss was measured in a single fibre drawn from the same Ge-doped preform with single mode core and cladding diameter of $100 \mu \mathrm{m}$. The loss profile along the length of the MEF was similar for all the fibre-elements. Also, no crosstalk (down to the level of $-80 \mathrm{~dB}$ ) was observed between the different fibre-elements when measured using a laser source at $1550 \mathrm{~nm}$.

The fibre-elements were subsequently connected in series with the output of one fibreelement spliced to the input of the next, thus obtaining an effective total transmission length of $28.5 \mathrm{~km}$. Fig. 3 compares the OTDR loss for one of the fibre-elements with the complete channel length obtained by looping back the fibre-elements.



Fig. 3 OTDR loss for one of the fibre-element of 3MEF from both ends

A transmission experiment was performed on the fibre using the experimental setup shown in Fig.4. F1, F2 and F3 represent the three different fibre-elements of the 3-MEF. Twenty one 10-Gbps-OOK channels, two 40-GbpsBPSK channels, and two 24-Gbps-QPSK channels were wavelength multiplexed into the fibre resulting in a data rate of $338 \mathrm{Gbps}$ per fibre-element and an overall transmission rate of 1014 Gbps. Prior to retrieving the channels at the output, dispersion compensating fibre (DCF) was used. Due to the limited choice of tailored DCF modules in our lab, $120-\mathrm{ps} / \mathrm{nm}$ of dispersion was left uncompensated.

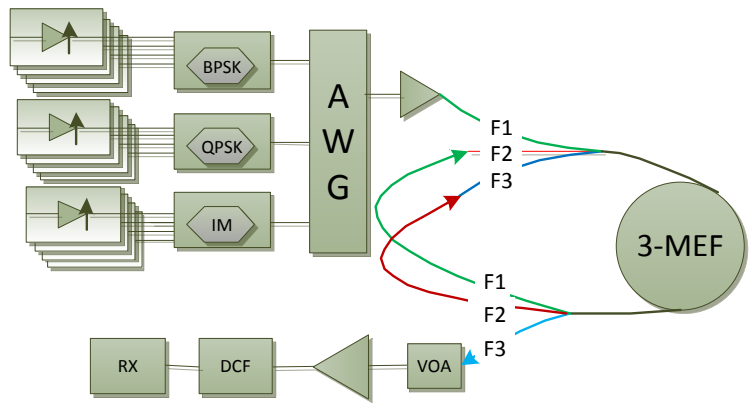

Fig. 4 Schematic setup for BER measurement

All the channels were detected to be error-free at the output. Indicatively, the bit-error-rate (BER) vs. optical-signal-to-noise ratio (OSNR) 
curves for three of the transmitted signals (one for each modulation format) are shown in Figs.5a-c. For reference, the wavelengths of the BPSK, OOK and QPSK channels shown in the figure were $1555.710 \mathrm{~nm}, 1541.804 \mathrm{~nm}$ and $1551.562 \mathrm{~nm}$ respectively. A slight difference in slope is evident for higher baud rate signal (BPSK) as compared to QPSK. It can be seen from Fig.5d that the input and attenuation compensated output spectra of the channels launched in the MEF overlapped, indicating no OSNR degradation.
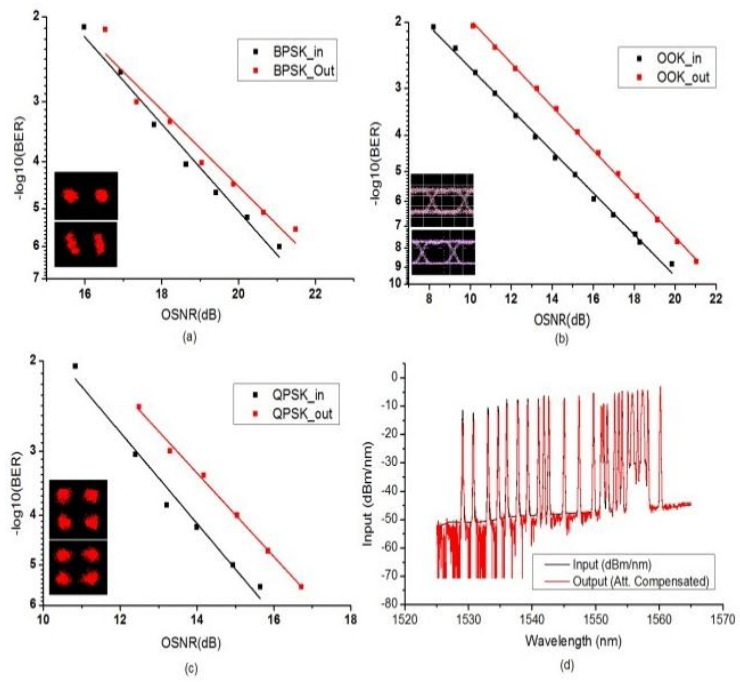

Fig. 5 BER vs. OSNR plots for a) 40-Gbps-BPSK, b) 10-Gbps-OOK, and c) 24-Gbps-QPSK channels. Inset shows constellation/eye diagrams at the input (top) and output (bottom), respectively; d) input and output spectra (attenuation-compensated) for all the channels in operation

Similar experiments were performed on a 3.07$\mathrm{km}$ length of compact 3-MEF with an overall coated diameter of $297 \mu \mathrm{m}$, which was fabricated from the same preform to increase the element density. Fig. $2 b$ shows the crosssection image of the compact 3-MEF. The compact 3-MEF was also measured to be free from any crosstalk, and its performance was again confirmed by performing BER measurements. The same setup of Fig. 4 was used and this time the overall length was $9.1 \mathrm{~km}$. It was noted however, that the loss of this $3-M E F$, as measured at $1550 \mathrm{~nm}$ using an OTDR was significantly higher for two of the fibre-elements than it was in the third $(2.5 \mathrm{~dB} / \mathrm{km}$ as compared to $\sim 0.7 \mathrm{~dB} / \mathrm{km}$ ). We believe that this was due to imperfect coating with the primary polymer resulting from the limited choice of primary coating dye sizes currently available to us. A $190 \mu \mathrm{m}$ primary dye was used for the compact 3-MEF fabrication, which was comparable to a notional triangular geometry fibre diameter of $173 \mu \mathrm{m}$. As seen in fig. $2 \mathrm{~b}$, the fibre-elements were positioned off-centered with respect to the outer coating. This problem should be readily resolved by further refining the MEF drawing process.

\section{Conclusions}

MEFs have been presented as a promising candidate for SDM implementation. Their main advantage is that they remove the constraint in the maximum cladding size that exists in MCFs, allowing an increase in the potential number of cores without compromising crosstalk. In addition, MEFs do not require the development of any MUX/DEMUX device technologies for access to the cores. In the future, we envisage that the MEF and MCF approaches could even be combined to achieve even higher numbers of cores per-fibre. For example, a 7-element MEF (7-MEF) comprising 3-core MCFs as their fibreelements would allow multiplexing of 21 cores. Smaller fibre element sizes should also be possible.

Compact and non-compact 3-MEFs from Ge doped preform exhibited crosstalk-free operation. In both fibres, all of the 25 channels contributing to $1014 \mathrm{Gbps}$ data rate transmission experiments were retrieved errorfree for effective transmission lengths of $9.21 \mathrm{~km}$ and $28.5 \mathrm{~km}$ respectively. Also, the fibre passed successfully through a 400-kpsi proof strength.

\section{Acknowledgements}

This work was supported by the UK Engineering and Physical Sciences Research Council grant EP/I01196X/1 (Hyperhighway). The authors would like to thank Sumiaty Ambran and Reza Sandoghchi for extending their help with MEF microscope image and OTDR measurement respectively.

\section{References}

[1] R. J. Essiambre, and R. W. Tkach, in Proc. IEEE, Vol. 100, No. 5, 1035-1055 (2012)

[2] R. J. Essiambre, and P. Winzer, J. Lightwave Tech., Vol. 28, No.4, pp. 662, (2010)

[3] A. D. Ellis, J. Zhao, and D. Cotter, J. Lightwave Tech., Vol. 28, No. 4, pp. 423, (2010) [4] V. Sleiffer et. al., in ECOC, OSA Technical Digest (online), Th.3.C.4, (2012)

[5] H. Takara et. al., in ECOC PDP, Th3.C.1, (2012)

[6] J. Sakaguchi et. al., in OFC, OW11.3, (2013)

[7] S. Matsuo et. al., Opt. Letters, Vol. 36, No. 23 (2011)

[8] S. Jain et. al., Opt. Letters, Vol. 38, No. 4, (2013) 